\title{
Effective Doppler Mitigation in Critical Satellite Communications
}

\author{
Alessio Fanfani, Simone Morosi ${ }^{(凶)}$, Luca Simone Ronga, and Enrico Del Re \\ Information Engineering Department (DINFO), University of Florence, \\ Via S. Marta 3, 50139 Florence, Italy \\ \{alessio.fanfani, simone.morosi, enrico.delre\}@unifi.it, \\ luca.ronga@cnit.it
}

\begin{abstract}
The modems for telemetry and telecommand applications are a key component in each satellite system: they shall guarantee reliable and effective performance during every mission phases including also critical scenarios such as the control operation of the satellite while it is placed into its orbit, the disposal of a satellite at the end of its life or the deep-space missions. In these scenarios, the link could be unstable and with a rapid variability and the communication become bursty and be characterized by poor performance. This paper introduces an alldigital implementation of a receiver, which is based on the Differential PSK (D-PSK) modulation and an enhanced version of the Digital Delay and Multiplier frequency estimator and compensator for mitigating the Doppler effect, and that results to be perfectly compliant with the aforementioned requirements. The performance of the proposed receiver is extensively studied and compared with an incoherent technique which is based on the Double Differential PSK (DD-PSK) modulation and is known to be suitable for sat-com in critical scenarios.
\end{abstract}

\section{Introduction}

The goal of the development of advanced heterogeneous satellite systems $[1,2]$ requires innovative and robust technological solutions. Telemetry and telecommand (TM/TC) systems along with electrical power, on-board data handling and attitude and determination control modules are essential for a spacecraft. The reliability and robustness are key features for a satellite communication system, particularly during critical satellite mission phases, like the early operations after separation from upper launcher stages or the end of life manoeuvres $[3,4]$. In these scenarios, the spacecraft may have reduced functionalities and uncontrolled attitude and consequently, the communication link could be weak, unstable and with a rapid variability e.g., signal's amplitude could drop down or go up according to satellite tumbling rate $[5,6]$. In this context, the communication becomes bursty and the link reliability harms the bit rate performance.

This paper proposes an advanced radio receiver for Low Earth Orbit (LEO) satellite communication system. The proposed modem is based on a robust and efficient frequency compensator to mitigate the Doppler effect, which is one of the main channel impairments in a LEO mission. 
The problem of Doppler compensation has been deeply discussed in literature $[8,16]$. Non coherent techniques are attractive due to their simple architecture; an example is the differential PSK (DD-PSK) that does not require a reference carrier since it performs the demodulation by processing the phase of the received signal in two successive intervals and estimating the relative difference $[9,10]$.

On the contrary, coherent techniques guarantee a better performance but are based on complex architectures because all the communication channel parameters (channel delay, frequency offset and phase) must be evaluated before the bit decision [11]. For instance, a receiver for LEO mission needs a frequency recovery circuit to reduce the frequency offset due to Doppler within the acquisition range of the carrier recovery. A typical frequency recovery system performs two functionalities, namely the estimate of the carrier frequency offset and the compensation by counter-rotating.

In the case of large frequency shift, an open-loop maximum likelihood (ML) scheme which is called the Delay and Multiply Method has been proposed in [7]. This method is suitable for burst communications thanks to the very short acquisition time; moreover, it is characterized by a mean square error that is comparable with the one of the closed-loop techniques [8]. Open-loop ML schemes only need one observation time to get the estimation whereas closed-loop require up to 5 observation times to complete the acquisition $[12,13]$.

Because of these interesting features, the Delay and Multiply estimator has been selected for our receiver. The proposed solution is also influenced by the idea of using a coarse and a fine estimation sequentially as used in the receiver realization described in [14].

The features of a receiver which is based on Delay and Multiply (D\&M) estimator will be compared with a receiver implementing an incoherent Double Differential PSK whose performance and implementation have been described in a previous contribution [5].

The paper is organized as follows: Sect. 2 provides an overview of the application's scenario including a characterization of the Doppler shift and of the link budget in a critical scenario. Section 3 proposes a complete description of the modem architecture and an analytical analysis of its operative principles Sect. 4 compares the performance of the whole receiver architecture with the one proposed in [5]. Finally, conclusive remarks are given in Sect. 5 .

\section{Channel Characterization}

\subsection{Doppler Effect}

One of the main channel impairments in TM/TC link for a satellite placed in non-geostationary orbit is the large and time-variant Doppler shift within a visibility window of satellite. 


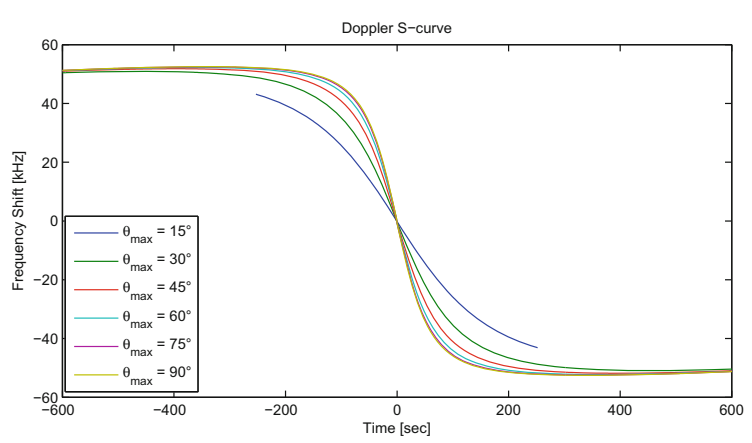

Fig. 1. Doppler shift S-curve

The Doppler Effect is caused by the relative motion of the satellite with respect to the ground station. It mainly depends on satellite orbit, ground station position (latitude) and the frequency value of the link.

The characterization of Doppler shift is a well debated argument in literature. In [15] I. Ali et al. show an exhaustive explanation of Doppler equations that are used in next sections to compare the performance of different receivers in the case of variable Doppler shift.

The frequency shift is represented by the S-curve, which is shown in Fig. 1. The shift is function of the time and of the maximum elevation angle $\theta_{\max }$. The shift is equal to zero in the middle of the visibility window when the elevation angle has the maximum value. The maximum shift is within $\pm 60 \mathrm{kHz}$ and it occurs when the elevation angle approaches the minimum elevation angle which is evaluated equal to $10^{\circ}$.

\subsection{Link-Budget}

A communication system for satellite TM/TC applications usually requires low or medium channel capacity. A bit rate of about $32 \mathrm{kbps}$ is enough for a Satellite Ground Operator to control and monitor the satellite within a visibility window. The operative frequency is in S-Band: in particular ITU radio regulation and satellite standard [17] reserves the following sub-ranges:

- Frequency range: 2025-2110 MHz for Earth to space link;

- Frequency range: 2200-2290 MHz for space to Earth link.

The satellite is usually equipped with a hemispherical patch antenna that is dedicated to the TM/TC link and placed on yaw satellite's face. When the satellite correctly points to Ground, the received power is easily computed by means the link budget equation; a detailed case study is described in [5].

An example of signal to noise ratio $E_{b} / N_{0}$ for a TM/TC link within a visibility window is represented by the curves in Fig. 2. The minimum $E_{b} / N_{0} \simeq 14 \mathrm{~dB}$ is in the downlink curve at the beginning and end of each visibility window, 


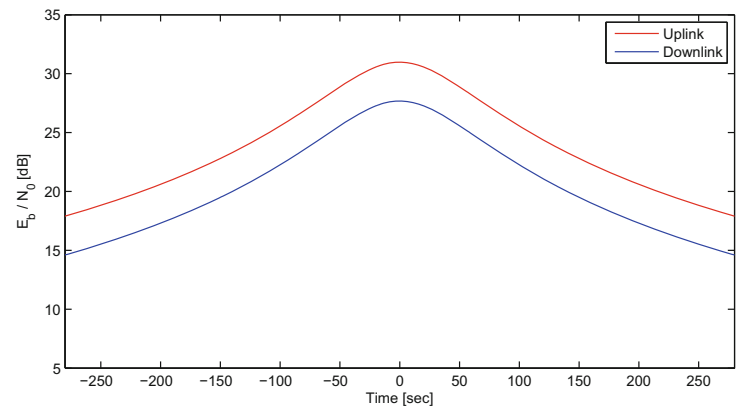

Fig. 2. Link budget curve in stable attitude condition

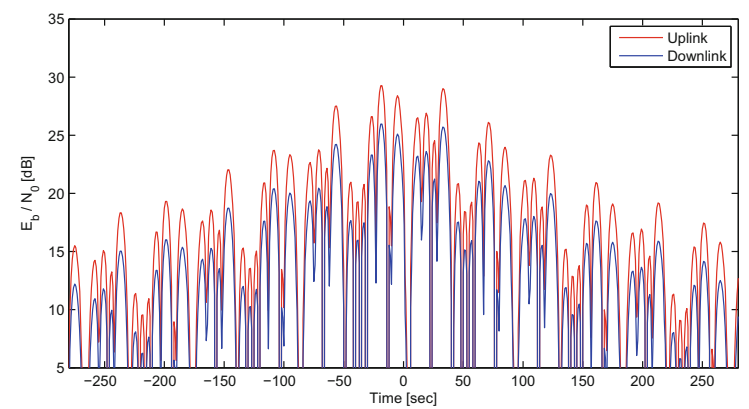

Fig. 3. Link budget curve at the satellite's end of life

when the satellite is closer to the horizon. Nevertheless, in a scenario with damaged attitude control system, the channel results becomes extremely unstable and the signal strength would follow the antenna radiation pattern dropping out completely when the antenna rotates away from ground station [6]. Figure 3 reports the values of the previously considered curves as given by the link budget curves in a possible critical scenario. A high tumble rate has been supposed around all satellite's axes. The presumed angular rotation velocities are $4 \mathrm{deg} / \mathrm{s}$ around Pitch axis, $14 \mathrm{deg} / \mathrm{s}$ around Roll axis and $10 \mathrm{deg} / \mathrm{s}$ around Yaw axis. A sequence of impulses underline the signal instability and an adequate signal power is obtained only in short burst intervals whose duration is often less than few seconds.

\section{Receiver Architectures}

The following section introduces the receiver architecture, that is oriented to a Software-Defined Radio (SDR) realization. A simulated implementation and a performance evaluation of the architecture have been performed in Simulink.

The receiver that is based on the use of the Digital D\&M estimator and compensator is a direct-conversion receiver [7], also known as zero-Intermediate 
Frequency (zero-IF) receiver: as it is known, it allows the Radio Frequency (RF) signal to be demodulated by a local oscillator whose frequency is as close as possible to the carrier frequency $f_{c}$, of the received signal.

The strategy which is pursued by the receiver is to estimate the Doppler frequency shift by evaluating the average frequency of the zero-IF input signal. In fact, that signal is frequency shifted away from zero hertz by the Doppler effect.

The implementation and the simulations that are described in this paper are based on the following assumptions:

- the signal delay and the clock recovery are performed before the carrier synchronization. The symbol delay is assumed zero;

- the pulse shape is an ideal rect and satisfies the Nyquist criterion for zero interference;

- the carrier $f_{c}$ is a deterministic parameter;

- the arbitrary phase shift is a uniformly distributed random variable;

- the channel is non frequency selective and has a flat frequency response.

\subsection{D\&M Estimator and Compensator}

The D\&M Estimator, that is represented in Fig. 4, matches to the phase variation to determine the instantaneous frequency of the input signal. The rate of change is computed by using the Euler's method of approximating differential equations. The instantaneous phase is generated by the arctangent function ( $\arg$ ) of the complex-valued input signal $x(t)$. The delay block $\Delta T$ which is used to compute the discrete differential is equal to the sampling time and $T_{0}$ is the symbol period. The block indicated with the ()$^{*}$ symbol represent the complex conjugate operator.

The analytical operating principle of the D\&M Estimator is defined by the following equations:

$$
\widehat{\Delta f}=\frac{1}{2 \pi \Delta T} \arg \left\{\int_{0}^{T_{0}} z(t) d t\right\}
$$

where:

$$
z(t)=x(t) x^{*}(t-\Delta T)
$$

The output signal $w(t)$ which is produced by the Direct Digital Synthesizer (DDS) has the form:

$$
w(t)=A \exp (-j(2 \pi \widehat{\Delta f} t))
$$

and the acquisition range of the estimator is equal to $\frac{1}{2 \Delta T}[7]$. 


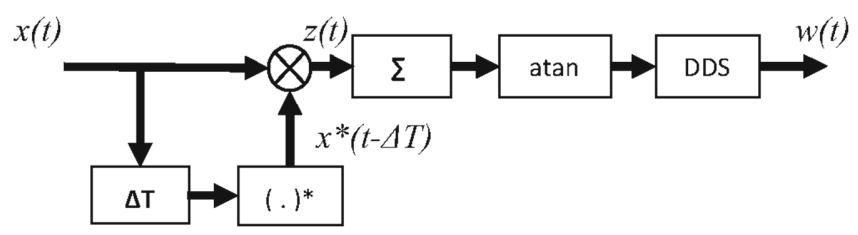

Fig. 4. D\&M estimator and compensator

\subsection{Baseband Decoder Block}

The base band section is based on the scheme of the D-BPSK demodulator that is depicted in Fig. 5.

The performance of the Delay and Multiply scheme depends on the Frequency value of the input signal: the greater the Frequency, the greater the variance of the estimation. For this reason, the receiver uses two Delay and Multiply estimators: The first D\&M is indicated as A and is a "coarse" estimator working on $r(t)$ signal: it performs a rough estimation of the frequency shift $\widehat{\Delta f}{ }_{A}$. The second one is defined as D\&M B: it is a "fine" estimator working on $e(t)$ signal, that is the signal with a frequency shift which is equal to the estimation error of D\&M A. Thanks to this mechanism the total estimator has a good performance also with large frequency shift of the input signal.

The $r(t)$ signal, that is affected by the Doppler shift is mixed with the compensation signals in order to translate the baseband message signal spectrum closer to the zero hertz value. After the Frequency compensation, an Integrate and Dump and a D-BPSK decision complete the receiving chain. The receiver doesn't need phase recovery scheme before the bit decision because the transmitted signal $r(t)$ is D-PSK modulated.

The input signal is defined by the following equation:

$$
r(t)=A \exp \left(j\left(2 \pi \Delta f t+\theta_{n}+\varphi_{n}\right)+n(t)\right.
$$

where:

- $\varphi_{n}$ is the differentially coded modulation information;

$-\Delta f_{D_{n}}$ is the Doppler shift on n-th symbol;

$-\theta_{n}$ is the phase error on $n$-th symbol.

- $\varphi_{n}$ is the phase contribution associated to the transmitted data, $\varphi_{n}=n k \pi \rightarrow$ $n \in Z, k=[0,1]$;

The $x(t)$ signal, that is obtained after frequency compensation and filtering, is equal to:

$$
\begin{aligned}
x(t) & =A \exp \left(j\left(2 \pi\left(\Delta f-\widehat{\Delta f}{ }_{A}-\widehat{\Delta f} f_{B}\right) t+\theta_{n}+\varphi_{n}\right)+n(t)\right. \\
& =A \exp \left(j\left(2 \pi\left(\theta_{n}+\varphi_{n}\right)+n(t)\right)\right.
\end{aligned}
$$

The performance of the Digital Delay-and-Multiply estimator is demonstrated through a complete simulation of the receiver performed in Simulink. 


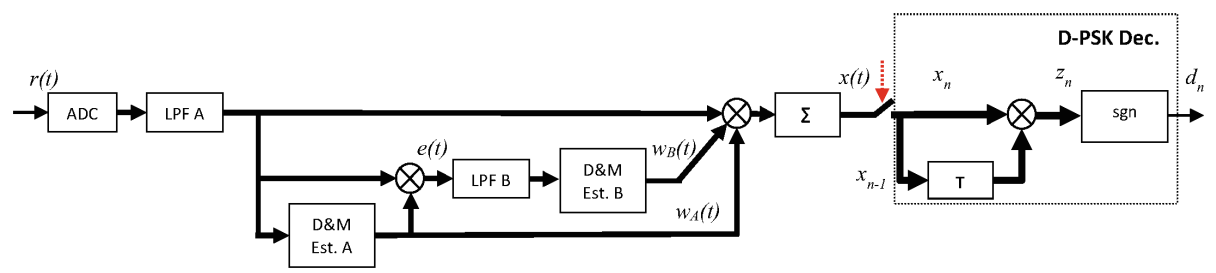

Fig. 5. Baseband decoder block diagram

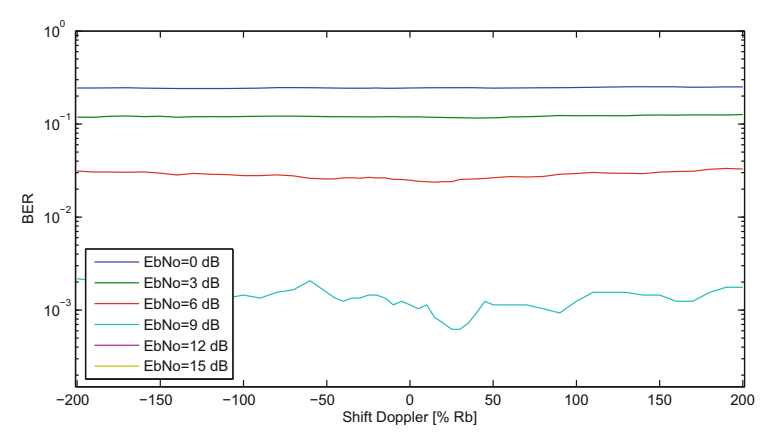

Fig. 6. Digital D\&M receiver performance

The curves in Fig. 6 show the bit error rate value as a function of the Doppler shift. The results show a constant BER and proves the effectiveness of the proposed solution. When the $E_{b} / N_{0}$ is greater than $12 \mathrm{~dB}$ the Bit Error Rate is lower than $10^{-4}$.

\section{Performance Comparison}

The previous section has shown the reason why a receiver that is based on the D\&M estimator is robust against the Doppler shift. In this section the performance of the proposed receiver will be compared with those obtained by DDPSK [5]. The performance are evaluated in three different scenarios. The first one, which is called fixed Doppler, is an ideal scenario with constant frequency Doppler shift, i.e., no time variation is assumed. The second scenario simulates a link that is affected by a real Doppler shift as defined in Sect.2.1. Finally, the last scenario takes into account, in addition to the Doppler shift, an extremely variable Signal to Noise ratio due to the satellite tumbling as depicted in Fig. 3.

\subsection{Fixed Doppler}

The bit errors rate curves are nearly constant for different Doppler conditions but the DD-PSK Bit Error Probability is much higher than the other. The Fig. 7 clearly highlights a better performance of the receiver based on Delay and Multiply estimation within the assumption of time-invariant frequency Doppler shift. 


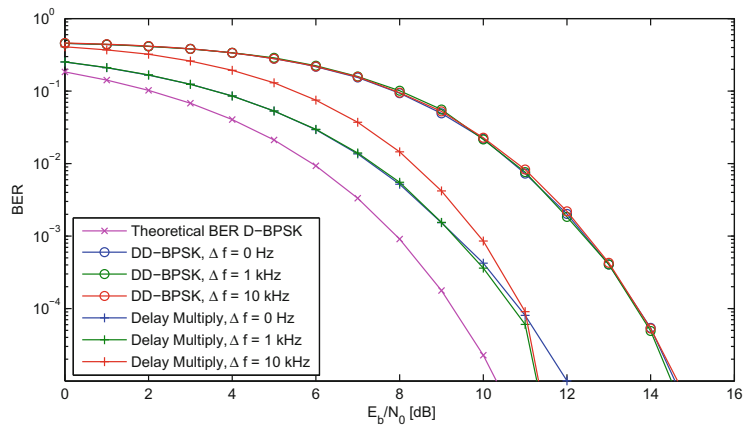

Fig. 7. Bit error rate for different modulation

The presented performance could be further improved by introducing efficient channel coding techniques.

\subsection{Variable Doppler}

This scenario considers a Doppler shift corresponding to the S-Curve in Fig. 1 with a maximum elevation angle $\theta_{\max }$ equal to $90^{\circ}$ and a minimum elevation angle equal to $20^{\circ}$. The visibility windows duration is $560 \mathrm{~s}$ during which are transmitted about 18350080 bit.

The selected S-curve is the worst operative case because it is characterized by the greatest Doppler shift Rate in the centre of the visibility windows.

The performance of both the receiving schemes is measured in terms of number of bit errors during a satellite visibility window. The curves in Fig. 8 shows the cumulative distribution of bit errors for different values of Signal to Noise ratio. The graphs confirms that the receiver based on Delay and Multiply frequency compensator has a very robust performance. That receiver has a constant bit error rate during all the visibility time; otherwise the DD-PSK has performance that depends on the Doppler shift Rate. Indeed, the greater growing rate

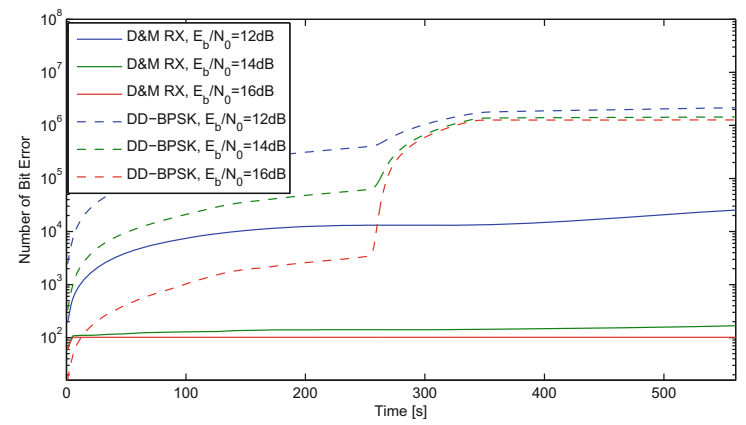

Fig. 8. Cumulative bit error for different modulation 
Table 1. Bit error rate with time variant Doppler shift

\begin{tabular}{l|l|l}
\hline$E_{b} / N_{0}, \mathrm{~dB}$ & BER of D\&M receiver & BER of DD-PSK receiver \\
\hline 12 & $1.46 \cdot 10^{-3}$ & $12 \cdot 10^{-2}$ \\
\hline 14 & $9.43 \cdot 10^{-6}$ & $7.9 \cdot 10^{-2}$ \\
\hline 16 & $5.61 \cdot 10^{-6}$ & $6.98 \cdot 10^{-2}$ \\
\hline
\end{tabular}

of the cumulative distribution is obtained for the maximum value of Doppler Rate. In Table 1 the average bit error rates are summarized for different values of Signal to Noise ratios that are computed at the end of a satellite visibility windows. In the case of receiver based on Delay and Multiply frequency compensator the BER performance that is obtained for the variable Doppler shift are similar to the ones of the constant Doppler scenario. The D\&M BER curves show an initial step due to the wrong bit decoding during the first observation time of the D\&M estimator.

\subsection{Tumbling Scenario}

In order to complete the comparison, the scenario with a tumbling satellite has to be considered. The results that are shown in Fig. 9 still prove the effectiveness of the receiver based on the D\&M. The curves represent the Bit Error Rate over the visibility windows for both the receivers. As expected, the BER is very high when the $E_{b} / N_{0}$ is poor, but as soon as the signal quality increases, the performance of D\&M is better than the one of the DD-PSK.

Also this graph confirms that the DD-PSK solution is weak when the Doppler rate is high: particularly, in the center of the visibility windows, the DD-PSK BER values are about $50 \%$.

The results of the simulation become clearer if, in the BER evaluation, only the bits with an acceptable signal to noise ratio are considered, i.e. when $E_{b} / N_{0}$ is greater than $12 \mathrm{~dB}$. In this case, the simulation shows that the BER of the

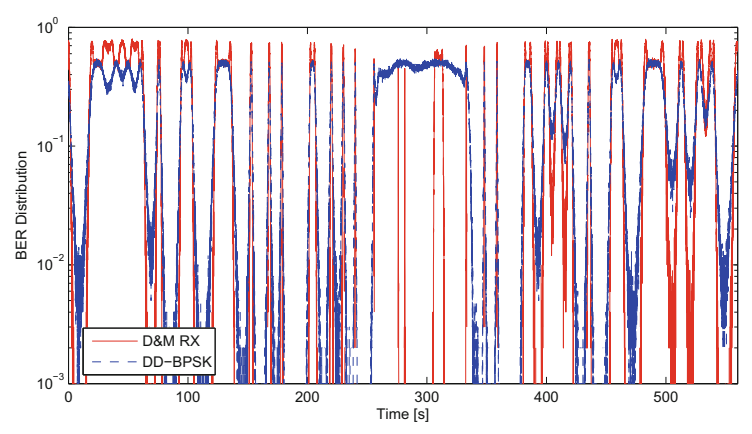

Fig. 9. Bit error rate over the time for different modulation 
receiver which is based on D\&M is equal to $8 \cdot 10^{-5}$ against a BER of the DD-PSK equal to 0.1 .

\section{Conclusion}

Space applications, such as the satellite decommission, have recently requested reliable and robust telecommunication systems in order to guarantee TM/TC communications also in emergency scenarios. This paper provides a possible channel model and proposes a possible transceiver implementation for these applications that is compared to different solutions.

The solution which is based on a frequency estimator and compensator named D\&M has a more complex architecture than the DD-PSK receiver but allows a very good performance. The results shows that D\&M receiver guarantees a reliable link also with fast channel variations and strong Doppler shift as shown in the paper. Therefore, the proposed D\&M receiver is a suitable implementation for communication in emergency scenario as the TM/TC for decommissioning device.

\section{References}

1. Del Re, E., Morosi, S., Ronga, L.S., Jayousi, S., Martinelli, A.: Flexible heterogeneous satellite-based architecture for enhanced quality of life applications. IEEE Commun. Magaz. 53(5), 186-193 (2015)

2. Del Re, E., Morosi, S., Jayousi, S., Sacchi, C.: Salice satellite-assisted localization and communication systems for emergency services. In: Proceedings of the 1st International Conference on Wireless Communications, Vehicular Technology, Information Theory, Aerospace and Electronic Systems (WVITAE), Aalborg, Denmark (2009)

3. IADC Space Debris Mitigation Guidelines (2002)

4. European Code of Conduct for Space Debris Mitigation: Issue 1.0 (2004)

5. Del Re, E., Fanfani, A., Morosi, S., Ronga, L.S.: Robust modem design for satellite communications in emergency scenarios. In: 2014 7th Advanced Satellite Multimedia Systems Conference and the 13th Signal Processing for Space Communications Workshop (2014)

6. Bruzzi, J.R., Jensen, J.R., Fielhauer, K.B., Royster, D.W., Srinivasan, D.K.: Telemetry recovery and uplink commanding of a spacecraft prior to three-axis attitude stabilization. In: Proceedings of the 2006 IEEE Aerospace Conference (2006)

7. Mengali, U., D'Andrea, A.N.: Synchronization Techniques for Digital Receivers. Springer, Heidelberg (1997)

8. Classen, F., Meyr, H.: Two frequency estimation schemes operating independently of timing information. In: Conference Recreation (GLOBECOM 1993), Houston, TX (1993)

9. Yuce, M.R., Wentai, L., Damiano, J., Bharath, B., Franzon, P.D., Dogan, N.S.: SOI CMOS implementation of a multirate PSK demodulator for space communications. IEEE Trans. Circ. Syst. I Regul. Papers 54(2), 420-431 (2007) 
10. Ma, C., Wang, D.: The performance of DDPSK over LEO mobile satellite channels. In: Proceedings of the 2000 Asia-Pacific Microwave Conference (2000)

11. Gardner, F.: Hangup in phase-lock loops. IEEE Trans. Commun. 25, 1210-1214 (1977)

12. Mengali, U., Morelli, M.: Data-aided frequency estimation for burst digital transmission. IEEE Trans. Commun. 45(1), 23-25 (1997)

13. Fitz, M., Lindsey, W.: Decision-directed burst-mode carrier synchronization techniques. IEEE Trans. Commun. 40, 1644-1653 (1992)

14. van der Westhuizen, E., van Rooyen, G.: Baseband Carrier Recovery and Phase Tracking as a Doppler Compensation Technique for a zero-IF SDR (2009)

15. Ali, I., Bonanni, P.G., Al-Dhahir, N., Hershey, J.E.: Doppler Application In LEO Satellite Communication Systems. Springer, Heidelberg (2002)

16. Ah-Thew, G.P.: Doppler Compensation for LEO Satellite Communication Systems (1998)

17. ECSS-E-ST-50-05C Rev. 2: Radio Frequency and Modulation, ESA-ESTEC, October 2011 